\title{
Effectiveness of sealing active proximal caries lesions with an adhesive system: 1-year clinical evaluation
}

\section{Clarisse Abuchaim ${ }^{(a)}$ \\ Marina Rotta ${ }^{(b)}$ \\ Rosa Helena Miranda Grande ${ }^{(c)}$ Alessandro Dourado Loguercio(d) Alessandra Reis ${ }^{(d)}$}

(a) Professor, Pediatric Dentistry Department, School of Dentistry, University of Oeste de Santa Catarina, Joaçaba, SC, Brazil.

(b) Private clinician, Joaçaba, SC, Brazil.

(c) Associate Professor, Dental Materials Department, School of Dentistry, University of São Paulo, SP, Brazil.

(d) Adjunctive Professor, Department of Restorative Dentistry, School of Dentistry, Universidade Estadual de Ponta Grossa, PR, Brazil.

\section{Corresponding author:}

Alessandra Reis

Departamento de Dentística Restauradora, Faculdade de Odontologia, Universidade Estadual de Ponta Grossa

Av. General Carlos Cavalcanti, 4748

Ponta Grossa - PR - Brazil

CEP: 84030-900

E-mail: reisale@hotmail.com

Received for publication on Mar 25, 2010 Accepted for publication on May 10, 2010

\begin{abstract}
The objective of this study was to evaluate the effectiveness of a therapeutic sealant to arrest non-cavitated proximal carious lesion progression. The study population comprised 44 adolescents who had bitewing radiographs taken for caries diagnosis. Non-cavitated lesions extending up to half of dentin thickness were included in the sample. In the experimental group $(\mathrm{n}=33)$, the proximal caries-lesion surfaces were sealed with an adhesive (OptiBond Solo, Kerr) after tooth separation. The control group $(\mathrm{n}=11)$ received no treatment, except for oral hygiene instructions including use of dental floss. Follow-up radiographs were taken after one year and were analyzed in comparison with baseline radiographs. In a blind study setting, visual readings were performed by two examiners, blinded to whether the examined radiograph was baseline or follow-up, and whether it concerned a test or control lesion. The efficacy of sealing treatment was evaluated by the McNemar test (0.05). About $22 \%$ of the sealed lesions showed reduction, $61 \%$ showed no change and $16 \%$ showed progression. For the control lesions, the corresponding values were $27 \%, 36 \%$ and $36 \%$ respectively. The number of lesions that showed reduction and no changes were merged and therefore $83.3 \%$ of the sealed lesions and $63.6 \%$ of the control lesions were considered clinically successful. No statistical significance was detected $(\mathrm{p}>0.05)$. In the course of 1 year, sealing proximal caries lesions was not shown to be superior to lesion monitoring.
\end{abstract}

Descriptors: Dentin-bonding agents; Dental caries; Dental bonding; Clinical trial.

\section{Introduction}

Minimally invasive dentistry adopts a philosophy that integrates prevention, remineralization and minimal intervention for placing and replacing restorations. ${ }^{1}$ Minimally invasive dentistry reaches the treatment objective by using the least invasive surgical approach, with removal of the minimal amount of healthy tissues. Within this concept, contemporary protocols for the treatment of dental caries support the use of sealants, not only as preventive treatment for sound fissures, but also to arrest dental caries progression by sealing active occlusal and proximal caries lesions. ${ }^{2,3,4}$

Carious disease results from the imbalance between causal and protective factors, including bacterial infection and, therefore, restoration 
of the decayed tooth does not cure the disease. Initial lesions occur beneath the enamel surface and can, to a large extent, be repaired by re-establishing the balance between demineralization and remineralization. This can be achieved by preventing acids produced by bacteria from continuing with demineralization of the tooth structure. It has been hypothesized that sealing an existing lesion from contact with the oral fluids should lead to eventual reduction and even death of these organisms, thereby arresting the lesion progression. ${ }^{5}$

Although sealants have been successfully placed over non-cavitated caries lesions in occlusal surfaces, $2,6,7,8,9,10,11$ few short- and long-term clinical evaluations have been reported on the method for managing proximal caries lesions ${ }^{3,4}$ Therefore, the aim of this study was to evaluate the clinical performance of an adhesive system used as a sealant to arrest the progression of proximal caries lesions over a period of 1 year. The null hypothesis to be tested was that the two groups would have a similar performance in the course of 1 year.

\section{Materials and Methods Sample and selection of lesions}

The individuals (18 to 40 years old, average of 27 years old) were selected from among those coming to the School of Dentistry, University of Oeste de Santa Catarina, for a dental checkup. Approximately 230 individuals were examined in order to check whether they met the inclusion criteria. The individuals had to be healthy and the bitewing radiographs that were taken had to show at least one proximal caries lesion, as well as a neighboring tooth and visible dental biofilm next to the lesion. If the patients showed interest in participating in the study, each individual was required to sign a consent form. Participants with dental prosthesis, or glass ionomer cement restorations near the lesion were excluded from the study. This study was approved by the Ethics Committee of the Institutional Review Board of the University under protocol number 128/2005.

Standardized bitewing radiographs were taken with the help of an individual device made of selfpolymerized acrylic resin placed on the film holder (Jon, São Paulo, SP, Brazil), using Ektaspeed Plus
No. 2 film (Eastman Kodak, New York, NJ, USA) with an exposure of $70 \mathrm{kV}, 7 \mathrm{~mA}, 0.6 \mathrm{~s}$. Films were developed and fixed using a 9000 model automatic processor (Dent-X, New York, NJ, USA).

All proximal lesions shown on the bitewing radiographs of each individual were recorded and scored according to the following system: (1) radiolucency restricted to the outer half of the enamel; (2) radiolucency involving the inner half of the enamel; (3) radiolucency in the outer third of the dentin; (4) radiolucency in the outer half of the dentin and (5) radiolucency in the inner two thirds of the dentin. Only lesions with radiographic scores of 1, 2, 3 and 4 could be part of this study, as those with score 5 probably needed operative dentistry.

\section{Clinical procedures}

Patients in the control group were instructed on how to use dental floss (Colgate Total, Colgate, São Paulo, SP, Brazil) and brush their teeth with fluoridated toothpaste (Colgate Total 12 Clean Mint, Colgate, São Paulo, SP, Brazil). The same was done for patients in the experimental group; however they had proximal caries lesions sealed with a filled adhesive system (OptiBond Solo Plus, Kerr, Orange, CA, USA). The surface was cleaned with a pumice slurry and rubber cup and the lesion was isolated by placing rubber dam around the tooth to be sealed and the neighboring teeth. An elective temporary proximal space was created using a metal separator placed in the proximal space. As the metal separators and the dental clamps could injure the gingival tissue, the area was previously anesthetized. If this technical approach prevented the clinicians from obtaining sufficient clinical space, an elastic band was put into place and left for 24 hours before the sealing protocol.

The proximal surface of the carious lesion was etched for $20 \mathrm{~s}$ with a $35 \%$ phosphoric acid gel (3M ESPE, St. Paul, MN, USA). The adjacent surface was protected with Teflon tape. The etchant was thoroughly washed away with water, then the tooth was air-dried. When the proximal surface was completely dried, a two-step etch-and-rinse adhesive system (OptiBond Solo, Kerr, Orange, CA, USA) was slowly applied to the etched area in a very thin layer, using 
a very thin microbrush (FGM Dental Products, Joinville, SC, Brazil). A slight dry air stream was applied for $20 \mathrm{~s}$ to allow solvent evaporation; excess material was removed with unwaxed dental floss and the adhesive was light polymerized for $20 \mathrm{~s}$ at $500 \mathrm{~mW} /$ $\mathrm{cm}^{2}$ (VIP, Bisco Inc. Schaumburg, IL, USA). After polymerization, the sealant was inspected with a very thin explorer to check for complete coverage. Excess adhesive from non-etched areas was carefully removed with the same instrument. All participants in the study were then instructed to perform flossing of all proximal lesions every day, including the sealed surface.

The participants were instructed to continue their treatment at the Dental School, and provided with a letter asking the dentist not to treat the selected lesions without informing the investigator first. After 6 months and 1 year, the patients were recalled for the follow-up radiograph of control and test lesions.

\section{Radiographs interpretation}

The outcome variable was the caries progression status (regression, no change or progression) for the selected lesions after 1 year. The radiographs (baseline and 1-year) were read by 2 investigators by conventional visual paired reading. All radiographs were digitized at $600 \mathrm{dpi}$ (Scanjet 6100 scanner; Hewlett-Packard, Oregon, CA, USA) and stored in maximum-quality JPEG format.

The depth of each selected lesion was visually assessed on the baseline radiographs by an external examiner, based on the previously reported score system. For all visual readings (seen at x10 magnification on the computer screen, HP Pavillion Tx2000, Palo alto, CA, USA), the examiners were blinded to whether the examined radiographs were baseline or follow-up and whether they concerned test or control lesions. The examiners were asked to determine the progression status of the radiograph placed on the right against the radiograph placed on the left, with further randomization as regards the position (left/right) of baseline or follow-up images.

\section{Statistical analysis}

Intra-examiner reproducibility was measured after the two examiners re-read $100 \%$ of the paired radiographs 14 days after the first reading. Inter-examiner reproducibility was assessed after the first set of measurements. Both intra- and inter-examiner reproducibility was assessed using non-balanced Cohen's Kappa statistics. ${ }^{12}$ The caries progression status of the test and control lesions after 1 year was analyzed descriptively. The efficacy of the sealing procedure was finally evaluated by the McNemar test $^{13}$ at $95 \%$ confidence level.

\section{Results}

A total of 230 participants were examined to check whether they met the inclusion criteria. However, from among them, only 45 teeth were included in the study, 13 being in the control group and 32 in the experimental group. Three out of 45 teeth (2 from the control group and 1 from the experimental group) were excluded from the study due to difficulties with reading the radiograph. Therefore, after one year only 42 teeth were available for evaluation. In 10 teeth, an elastic band was used for tooth separation. Table 1 shows the distribution of selected lesions according to tooth type, surface and score. The majority of the test lesions were located in premolar teeth.

The inter-examiner reproducibility for the first set of paired readings was 0.85 and the intra-examiner reproducibility was 0.78 and 0.85 for examiners 1 and 2, respectively.

Graph 1 presents the outcome in terms of regression, no change and progression for the two groups after 1 year. In the experimental group, regression of the lesions was seen in $22 \%$ of the cases (7/31), no

\begin{tabular}{|c|c|c|c|c|c|c|c|c|c|c|}
\hline \multirow{3}{*}{$\begin{array}{l}\text { Table } 1 \text { - Distribution of } \\
\text { the lesions according to } \\
\text { tooth type, surface and } \\
\text { radiographic score. }\end{array}$} & \multirow{2}{*}{ Groups } & \multicolumn{3}{|c|}{ Tooth type } & \multicolumn{2}{|c|}{ Surface } & \multicolumn{4}{|c|}{ Score } \\
\hline & & Anterior & PM & M & Mesial & Distal & 1 & 2 & 3 & 4 \\
\hline & Experimental & - & 20 & 11 & 15 & 16 & 8 & 13 & 6 & 4 \\
\hline & Control & 1 & 8 & 2 & 6 & 5 & 3 & 3 & 3 & 2 \\
\hline
\end{tabular}




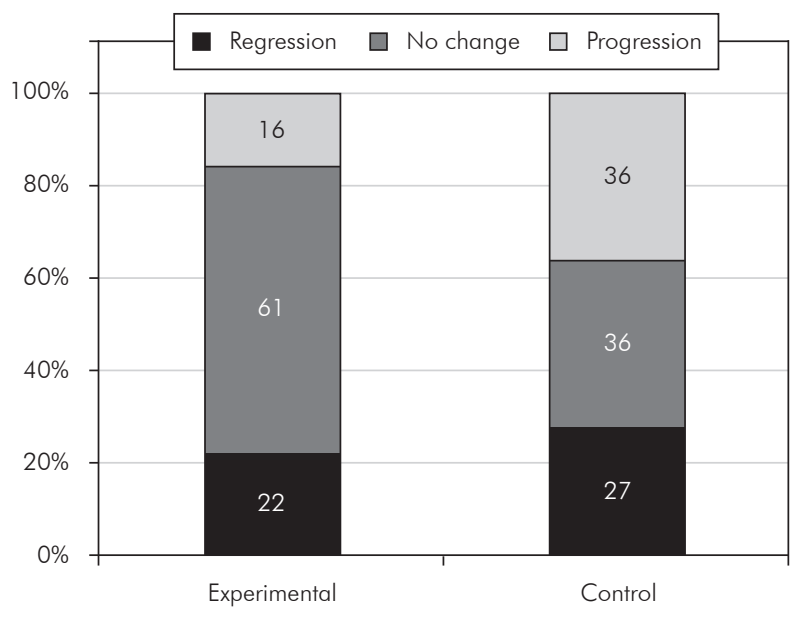

Graph 1 - Distribution of cases (\%) according to progression status of experimental and control lesions (regression, no change, progression).

change in $61 \%$ of the cases (19/31) and progression was detected in $16 \%$ of the cases (5/31). All lesions that showed progression were restored according to conventional restorative procedures and the other teeth are still being monitored. As regards the control group, regression, no change and progression were observed in $27 \%$ (3/11), 36\% (4/11) and 36\% $(4 / 11)$ of the cases, respectively. Figure 1 shows representative radiographs of caries lesions with progression, no change and regression.

Cases in which regression and no change of the lesions were detected after 1 year were considered clinically successful; that is $26 / 31(83.9 \%)$ of the cases in the experimental group and 7/11 (63.6\%) of the cases in the control group. This difference, however, was not considered statistically significant by the McNemar test ( $\mathrm{p}=0.12 ; 95 \%$ CI: $8-37 \%$ ).

\section{Discussion}

The present investigation did not detect any significant difference between the experimental and control groups, which is contrary to the findings of previous studies ${ }^{3,4}$ One of the reasons that might explain this lack of difference between the groups in the present study is the short follow-up period. Gomez et al. ${ }^{3}$ followed-up the participants over a 2-year period, while the follow-up by Martignon et al. ${ }^{4}$ was an 18 -month period.

It is known that the caries progression rate of proximal lesions is rather low, particularly in adult patients who have received regular fluoride treatment or who consume fluoridated water. ${ }^{14,15}$ Only $2 \%$ of the lesions located in dentin progressed radiographically to the inner half of dentin in a period of 20 months. ${ }^{16}$ A recent meta-analysis reported that the annualized probability of progression for nonsealed, non-cavitated lesions is only $12.6 \% .^{2}$

Although no significant difference was detected between the sealed and unsealed groups, a trend towards higher clinical effectiveness of the sealed lesions over unsealed lesions could be seen. Had a larger sample size been used, the difference could have been significant. Moreover, there is enough clinical evidence that states the effectiveness of this procedure both on occlusal ${ }^{7,8,10,11,17}$ and proximal surfaces. ${ }^{3,4}$ A recent review on the effect of dental sealants on the bacteria levels in caries lesions found that sealants significantly reduced bacteria levels in cavitated lesions, ${ }^{5}$ supporting the findings of a recent meta-analysis that sealants prevented caries progression. ${ }^{2}$ In the aforementioned meta-analysis study, the prevented fraction of lesion progression with the sealing protocol ranged from $61.6 \%$ to $100.0 \%$, with a median of $74.2 \% .^{2}$ In combination, these two sets of findings suggest that when sealants are retained, thereby blocking access to fermentable substrates, bacteria do not appear to be capable of exerting their cariogenic potential, even if low levels of bacteria still persist. ${ }^{5}$ Although observations in the aforementioned studies were made when a hydrophobic sealant was applied to caries lesions, one may hypothesize that the same assumptions are valid for sealing with dental adhesives. The authors of this study opted to use an adhesive system for proximal caries sealing due to 1 ) the lower viscosity of this material when compared with conventional sealants, which allowed easy handling within the proximal space; 2 ) better penetration of the adhesive system into natural lesions leaving no overhang that could bias the radiograph readings; ${ }^{4}$ and 3 ) the clinical success of using adhesive systems for sealing sound occlusal fissures. ${ }^{18}$

The application of an adhesive system or sealant on extracted human teeth with proximal non-cavitated enamel lesions may protect the surface against 


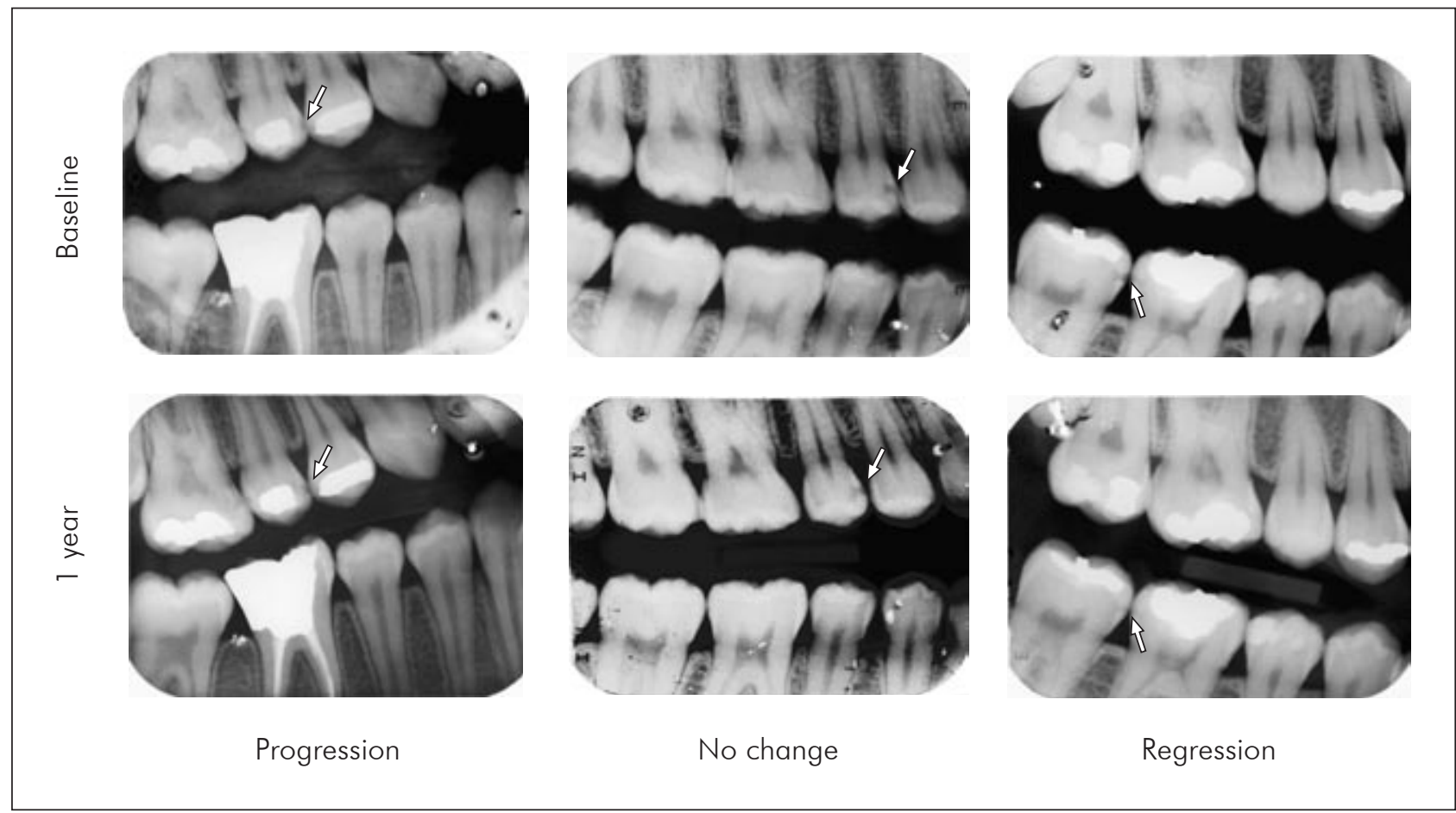

Figure 1 - Representative radiographs of caries lesions that showed progression, no change and regression.

exposure to acids produced by bacteria in the overlying plaque biofilm and also decrease the number of viable microorganisms in the lesion under the sealant. ${ }^{5}$ Studies have shown that the zone of artificial enamel lesions can be occluded by infiltration with polymeric materials. ${ }^{19,20}$ Gomez et al. ${ }^{21}$ reported that the application of a pit-and-fissure sealant on extracted human teeth in proximal non-cavitated enamel lesions infiltrated the superficial porous caries lesion and produced resin tags of up to 6 microns in length forming a physical barrier. This irregular pattern of infiltration could be attributed to the high viscosity of sealants making it difficult for them to penetrate into the microporosities of an enamel lesion. Future studies should be conducted in order to identify the best product for proximal sealing.

It is also worth mentioning that the effectiveness of this adhesive sealing technique is likely to be dependent on the quality of the material coverage over time. For instance, one study found that caries lesions measured by radiographic assessment were more likely to regress under intact sealants than under defective sealants. ${ }^{7}$

There are two technical aspects of the present study that differed from those of Gomez et al. ${ }^{3}$ and Martignon et al. ${ }^{4}$ The first was the use of rubber dam isolation in all cases in order to avoid contamination of the restorative field; and the second was the method used for tooth separation. While the previous authors used orthodontic elastic bands two days before the sealing procedure, in the present study, metal separators were used. Therefore only one clinical appointment was required for the sealing procedure. If this technical approach prevented the clinician from obtaining sufficient clinical space, an elastic band was placed and left for $24 \mathrm{~h}$ before the sealing protocol. However this was required in few cases, particularly when the tooth to be sealed was located in the mandible and between molars.

The therapeutic use of sealants for carious lesions is in line with strategies focused on a more tissue-preserving approach when restoring teeth. The concept of minimally invasive dentistry has focused on the maximum conservation of demineralized enamel and dentin. ${ }^{22}$ Although no significant difference could be observed in the present clinical setting, probably due to reduced sample size, the results show the potential of the therapeutic sealing 
technique to act as a non-invasive treatment of early proximal enamel lesions.

As pointed out by Gomez et al., ${ }^{20}$ arresting the lesion progression implies that cavity preparation for a restoration can be avoided, which prevents iatrogenic damage to the adjacent tooth, ${ }^{23,24}$ since $70 \%$ of the proximal Class II cavity preparations cause some damage to the adjacent tooth and this surface might develop a lesion more frequently than an undamaged surface. ${ }^{23}$ Another advantage of this technique is that placement of sealants is esthetically appropriate, inexpensive and can be repaired in case of failure without further removal of dental structure.

\section{References}

1. White JM, Eakle WS. Rationale and treatment approach in minimally invasive dentistry. J Am Dent Assoc. 2000 June; 131(Suppl):13S-19S.

2. Griffin SO, Oong E, Kohn W, Vidakovic B, Gooch BF, CDC Dental Sealant Systematic Review Work Group, et al. The effectiveness of sealants in managing caries lesions. J Dent Res. 2008 Feb; 87(2):169-174.

3. Gomez SS, Basili, CP, Emilson CC. A 2-year clinical evaluation of sealed noncavitated approximal posterior carious lesions in adolescents. Clin Oral Invest. 2005 Dec; 9(4):239243.

4. Martignon S, Ekstrand KR, Ellwood R. Efficacy of sealing proximal early active lesions: An 18-month clinical study evaluated by conventional and subtraction radiography. Caries Res. 2006 Oct; 40(5):382-388.

5. Oong EM, Griffin SO, Kohn WG, Gooch BF, Caufield PW. The effect of dental sealants on bacteria levels in caries lesions: a review of the evidence. J Am Dent Assoc. 2008 Mar; 139(3):271-278.

6. Going RE, Loesche WJ, Grainger DA, Syed SA. The viability of microorganisms in carious lesions five years after covering with a fissure sealant. J Am Dent Assoc. 1978 Sep; 97(3):455462 .

7. Handelman SL, Leverett DH, Solomon ES, Brenner CM. Use of adhesive sealants over occlusal carious lesions: radiographic evaluation. Community Dent Oral Epidemiol. 1981 Dec; $9(6): 256-259$.

8. Handelman SL, Leverett DH, Espeland M, Curzon JA. Clinical radiographic evaluation of sealed carious and sound tooth surfaces. J Am Dent Assoc. 1986 Nov; 113(5):751-754.

9. Heller KE, Reed SG, Bruner FW, Eklund SA, Burt BA. Longitudinal evaluation of sealing molars with and without incipi-

\section{Conclusions}

The therapeutic use of sealants for carious lesions is in line with strategies focused on a more tissuepreserving approach when restoring teeth. The use of adhesive systems to seal proximal caries lesions is a feasible technique. However, over the 1 year evaluation, therapeutic sealing and lesion monitoring were equivalent in terms of arresting lesions.

\section{Acknowledgements}

The authors of this study are very grateful to Dr. Priscila Bresciani, Dr. Bernardo Hahn and Dr. Franciele Loeblein for the help provided in the clinical part of this study.

ent dental caries in a public health program. J Public Health Dent. 1995 Summer; 55(3):148-153.

10. Handelman SL, Shey Z. Michael Buonocore and Eastman Dental Center: A historic perspective on sealants. J Dent Res. 1996 Jan; 75(1):529-534.

11. Mertz-Fairhurst EJ, Schuster GS, Fairhurst CW. Arresting caries by sealants: results of a clinical study. J Am Dent Assoc. 1986 Feb; 112(2):194-197.

12. Fleiss IL. Statistical methods for rates and proportions. 2nd ed. New York: Wiley; 1981. p 159-86.

13. Siegel S, Castellan NJ Jr. Nonparametric statistics for the behavioral sciences. Singapore: McGraw-Hill; 2002. The case of one sample, two measures or paired replicates. p. 73-101.

14. Shwartz M, Gröndahl HG, Pliskin JS, Boffa J. A longitudinal analysis from bite-wing radiographs of the rate of progression of approximal carious lesions through human dental enamel. Arch Oral Biol. 1984 Jul; 29(7):529-536.

15. Mejàre I, Stenlund H, Zelezny-Holmlund C. Caries incidence and lesion progression from adolescence to young adulthood: a prospective 15-year cohort study in Sweden. Caries Res. 2004 Mar-Apr; 38(2):130-141.

16. Pitts NB, Renson CE. Monitoring the behaviour of posterior approximal carious lesions by image analysis of serial standardized bitewing radiographs. Brit Dent J. 1987 Jan; 162(1):15-21.

17. Elderton RJ. Management of early dental caries in fissures with fissure sealant. Brit Dent J. 1985 Apr; 158(7):254-258.

18. Grande RH, de Lima AC, Rodrigues-Filho LE, Witzel MF. Clinical evaluation of an adhesive used as a fissure sealant. Am J Dent. 2000 Aug; 14(4):167-170.

19. Robinson C, Brookes SJ, Kirkham J, Wood SR, Shore RC. In vitro studies of the penetration of adhesive resins into artifi- 
cial caries-like lesions. Caries Res 2001 Mar-Apr; 35(2):136141.

20. Gomez S, Uribe S, Onetto JE, Emilson CG. SEM analysis of sealant penetration in posterior approximal enamel carious lesions in vivo. J Adhes Dent 2008 Feb; 10(2):151-156.

21. Gomez SS, Onetto JE, Uribe SA, Emilson CG. Therapeutic seal of approximal incipient noncavitated carious lesions: technique and case reports. Quintessence Int $2007 \mathrm{Feb}$; 38(2):99105.
22. Tyas MJ, Anusavice KJ, Frencken JE, Mount GJ. Minimal intervention dentistry--a review. FDI Commission Project 1-97. Int Dent J 2000 Feb; 50(1):1-12.

23. Qvist V, Johannessen L, Bruun M. Progression of approximal caries in relation to iatrogenic preparation damage. J Dent Res 1992 Jul; 71(7):1370-1373.

24. Lussi A, Kronenberg O, Megert B. The effect of magnification on the iatrogenic damage to adjacent tooth surfaces during class II preparation. J Dent 2003 May; 31(4):291-296. 